\title{
Performance of Players and Data Quality in a Citizen Science Casual Game
}

\author{
Igor Stankovic, $\mathrm{PhD}^{1}$, María Postigo Camps, $\mathrm{MSc}^{2}$, Daniel Cuadrado Sánchez, $\mathrm{MSc}^{2}$, \\ Miguel A. Luengo-Oroz, $\mathrm{PhD}^{2}$, Marisa Ponti, $\mathrm{PhD}^{1}$ \\ ${ }^{1}$ Department of Applied IT, University of Gothenburg, Sweden \\ ${ }^{2}$ Biomedical Image Technologies group, ETSI Telecomunicación, Universidad \\ Politécnica de Madrid, and Centro de Investigación Biomédica en Red en Bioingeniería, \\ Biomateriales y Nanomedicina (CIBER-BBN), Spain
}

\section{Abstract}

Background: Citizen science games are a type of Games with a Purpose (GWAPs), whose aim is to harness the skills of volunteers for solving scientific problems or contributing to action projects, where citizens intervene in social concerns. Employing games to collect data, classify images or even solve major scientific problems is a relatively new but growing phenomenon in citizen science. A main concern in citizen science is to ensure data quality. As games can be seen as having adverse effects on data quality, it is important to understand how citizen scientists produce data using games, how accurate this data can be, and whether and how games influence data quality.

Objective: The objective of this study was to evaluate the performance of individual players' data quality in MalariaSpot, a citizen science casual game in which volunteers are tasked with detecting parasites in digitized blood sample images.

Methods: We used descriptive statistics to analyze a subset of the gameplays recorded and stored in the MalariaSpot database, comparing its clicks to the Gold Standard position of the parasites. This subset includes 15,546 gameplays played over 38 known images that correspond to 97,200 clicks from 1,278 different players. Gameplays have been played via the Android and iOS applications and via the web version of the game. Images were acquired in three different locations and therefore sample preparation have been done by different lab technicians. Two distinct technologies were used for sample digitalization.

Results: The overall values for sensibility, specificity, and accuracy of the individual gameplays for the 38 images are $0.82,0.60$, and 0.29 respectively. High presence of parasites in an image makes it easier for players to detect them as their structures tend to look alike and can be compared. Being a simple casual game, the learning curve is very fast and after few minutes, players attend their typical performance level. Data quality is considerably lower in images acquired with mobile phones coupled to the microscope ocular compared to those digitized with standardized digitalization technologies.

Conclusions: The results indicate that data quality is influenced by the game, the technologies for image digitalization and the sampling preparation.

Keywords: crowdsourcing; malaria; image analysis; games for health 


\section{Introduction}

Citizen science games are a type of Games with a Purpose (GWAPs) [1], whose aim is to harness the skills of volunteers for solving scientific problems or contributing to action projects, where citizens intervene in social concerns. Citizen science is now an accepted term for a range of practices that involve members of the general public, many of which are not trained as scientists, in collecting, categorizing, transcribing or analyzing scientific data [2]. Successful citizen science projects include involving participants in classifying astronomical images, reporting bird sightings, counting invasive species in the field, using spatial reasoning skills to align genomes or folding proteins. These projects feature tasks that can only be performed by people (e.g. making an observation) or can be performed more accurately by people (e.g. classifying an image) with the support of computational means to organize these efforts. Citizen science games can be considered instances of human computation [3], because they can be used for tasks such as, for example, image recognition and problem-solving, which require human cognitive since automated data processing alone does not perform them reliably yet. Employing games to collect data, classify images or even solve major scientific problems is a relatively new but growing phenomenon in citizen science. The ultimate goal of using games in citizen science projects is often to get more from citizens: sustained engagement and retention of participants, more data, and/or higher quality data. Research into the use of games in citizen science is relatively novel but expected to grow rapidly, as the number of games in this area increases. Empirical studies have started to document the role of games to support citizen engagement and sustained participation (e.g., [4], [5]) and effect on data quality [6] and [7]. A main concern in citizen science is to ensure data quality, especially the intrinsic quality of data - that is, the accuracy and believability of the data provided by citizens [6]. Reasons for this concern include participants' lack of formal scientific training and limited scientific knowledge, uneven levels of expertise and anonymity, as well as nonstandardized and poorly designed methods of data collection [8]. It is important to understand how citizen scientists produce data using games, how accurate this data can be, and whether and how games influence data quality.

In this paper, we present an evaluation of the performance of individual players' in terms of data quality in MalariaSpot - a citizen science casual game in which volunteers are tasked with detecting parasites in digitized blood sample images coupled with a decision algorithm that combined the analyses from several players to produce an improved collective detection outcome [9]. Although individual performance of players (data quality) do not constitute the ultimate detection outcome in MalariaSpot - that combine the choices of different players into a single crowd decision- , it is nevertheless critical to understand them, as they constitute the "building blocks" of further processing and the collective parasite detection.

\section{Related Work}

\section{GWAPs, Citizen Science and Data Quality}

GWAPs support large-scale work by translating the required activity into elements of games [10]. GWAPs are now used in several citizen science projects in biomedical and clinical sciences. Applications like Foldit [20], EyeWire [21] and Phylo [22], to name three of the most well-known examples in biomedicine, use the engaging qualities of games to involve citizens to contribute their time and cognitive resources and solve computationally hard-to-automate tasks like protein folding, image recognition and 
gene annotation respectively. GWAPs are also seen as an attractive form of crowdsourcing because they do not have the inherent limits on capacity that are inherent to micro-task frameworks with per-work-unit costs [11]. The majority of games used in medical citizen science invite participants to perform microtasks [12]. Unlike megatasks that require a small number of talented and dedicated participants from a large pool of individuals, microtasks can be accomplished quickly and easily by anyone who can read simple instructions [13]. Microtask games require a large number of participants to process a huge volume of data in short time. Contributions are often aggregated to provide data quality as good as that provided by experts.

In relation to data quality, the framework adopted by [14] includes four dimensions: 1) intrinsic data quality, which refers to the believability or accuracy of data; 2) contextual data quality, which refers to timeliness, relevance and completeness of data; 3 ) representational data quality, which refers to how interpretable and easy to use data is, and 4) accessibility, which refers to how easy to access data is. Intrinsic data quality is a key concern in citizen science. Results from some previous studies indicate that observations from non-experts can be accurate compared to those from experts (e.g., [15] [16]). One reason for this high level of accuracy may be attributed to citizen scientists in the examined projects having expertise and a high level of interest in the topic [17]. Since in several citizen science projects, non-expert participants may not have such expertise, [14] argued that two important research goals in these projects include finding methods to turn scientific goals into tasks that members of the public can perform well, and finding methods - such as a gold standard data set already classified by experts - to confirm the quality of data provided by participants. In relation to the influence of games on the quality of data provided by citizen scientists, research appears to be still limited in volume and contexts, as few studies have been conducted mainly in taxonomic classifications of plants. For example, a classification game using taxonomic keys prepared by experts could produce usable data and could be a useful tool for scientists in the biological and life sciences [6], and quality of data in two games, one point-based and one story-based, was comparable and usable for scientific purposes [7].

\section{Materials and Methods}

\section{Description of the Game}

MalariaSpot is an online casual game with a medical purpose developed by a group of researchers at the Technical University of Madrid in Spain. The game aims to make malaria diagnosis more accessible in low-resource-settings regions, by decreasing time to diagnosis and economic limitations. Malaria diagnosis requires screening blood smears under a microscope to find and count malaria parasites on it. This task must be done by microscopists, who spend up to 20 minutes looking to each sample. There are currently 200 million new cases of malaria every year, $90 \%$ of them in low-income countries [18]. In some of these regions, there are not enough microscopists to analyze all the susceptible samples. By playing the game, non-specialist participants expect to help make malaria diagnosis more accessible and affordable for patients around the world.

The concept of the game is based on a microtask consisting in presenting images of blood smears to players and asking them to scan images by clicking on all the shapes that they think to be malaria parasites (Figure 1). In this manner, players achieve a score, the goal being to spot all the parasites in the image in a minute. The motto of the 
game is "a minute game against malaria." Players are only expected to have a good eye and be quick. No prior knowledge is required. The game shows how amateurs can tackle parasite counting over digital images. In [9], the authors reported that when utilizing an algorithm that combines the scores obtained by multiple non-experts playing in the same image, it is possible to get more than $99 \%$ accuracy. The same study reported that in April 2013, a year into the launch of the game, more than 15,000 people from 100 different countries generated almost one million clicks.

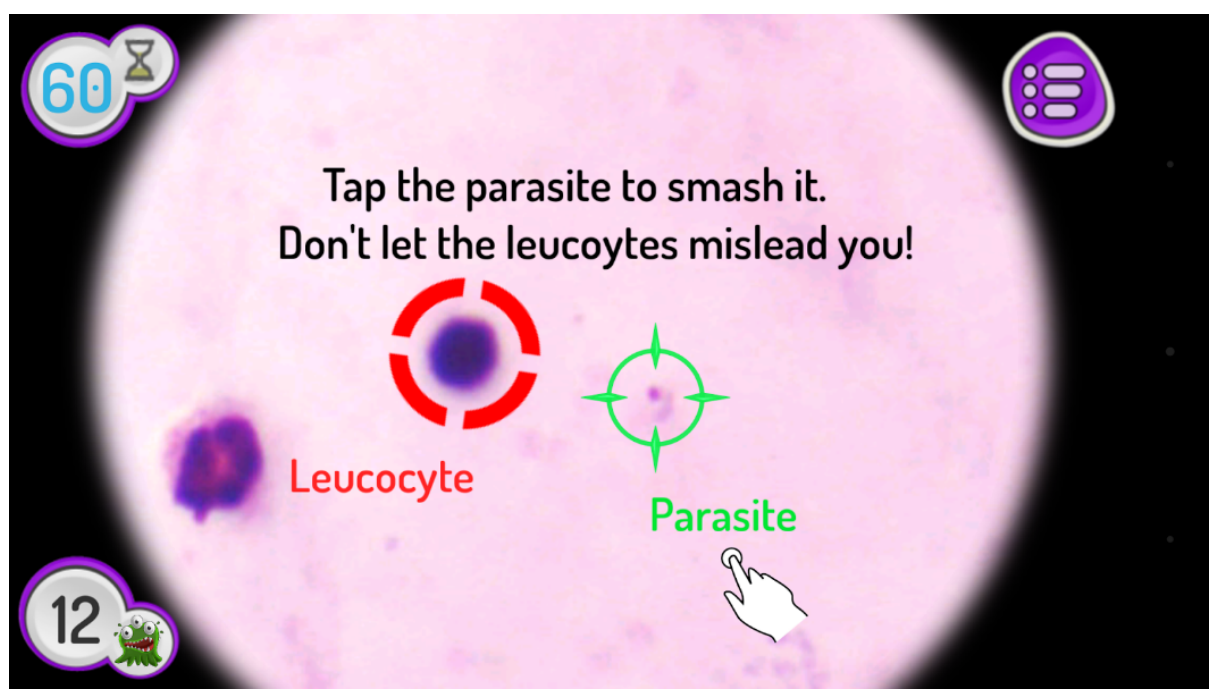

Figure 1. Screenshot from the Android application

MalariaSpot starts with a brief tutorial that shows players how malaria parasites look like. Then a player starts analyzing images, one per level. Each image corresponds to a quadrant of a sample and has to be analyzed in less than a minute. To move to the next level, a player should find at least $90 \%$ of the parasites in the image. If a player fails, he/she can start again from level 1 but with different images. The highest level reached was 385. Levels do not correspond to more difficult tasks/samples. The 'level' feature has been introduced in MalariaSpot for gamification purposes, since it gives players a sense of progression in the game. There are two types of images in MalariaSpot: 'known images' (also called 'Gold Standard images') and 'unknown images'. Known images have already been analyzed by experts and are included in a stored data set. Known images are used for training and quality control of players' performances. Unknown images have not been analyzed by experts and there is no prior knowledge of these images. These are the samples which need to be analyzed by players. Therefore, measuring performance is impossible for unknown samples and players move on to the next level regardless of their performance.

\section{Method}

We analyzed a subset of the gameplays recorded and stored in the MalariaSpot database, comparing its clicks to the Gold Standard position of the parasites. This subset includes 15,546 gameplays played over 38 known images that correspond to 97,200 clicks from 1,278 different players. Gameplays have been played via the Android and iOS applications and via the web version of the game. Out of the 38 known images, 25 images were taken in South Africa, eight were taken in Spain and five were taken in Mozambique. The images from South Africa were acquired with a standardized digitalization technology with a camera integrated into the microscope, while the 
images from Spain and Mozambique were acquired with a mobile phone camera coupled to the microscope objective (Figure 2).
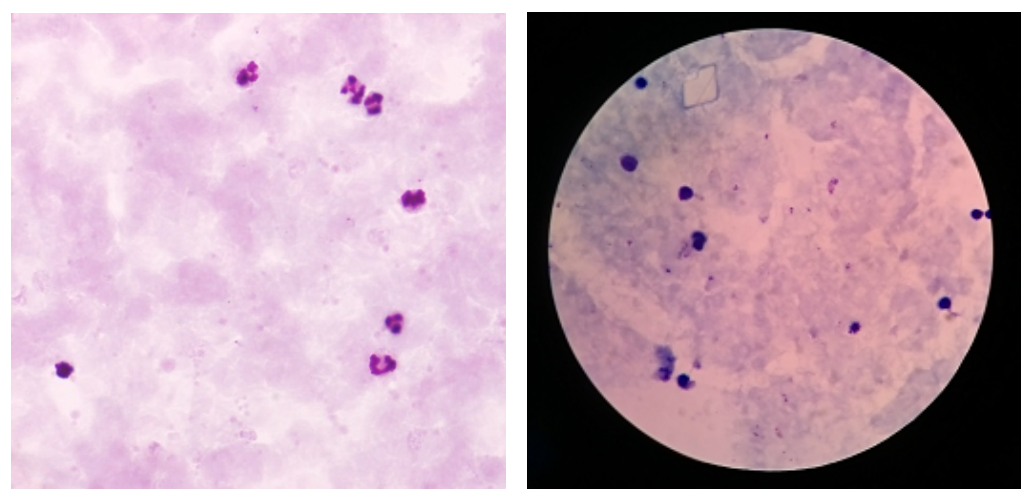

Figure 2. Image digitalized with a standardized technology (left) and image digitalized with a mobile phone (right).

Our data analysis was based on descriptive statistics of players' data using MATLAB and Microsoft Office Excel. Instead of performing the collective analysis on multiple players as performed by [9], we evaluated individual player performance. To analyze the data quality of each gameplay, we used three parameters: sensibility, specificity and accuracy. Data quality is directly proportional to the three parameters. True positives (TP) is the number of parasites detected by the player which are also present in the gold standard made by specialists and false positives (FP) is defined as the clicks made in the sample that do not correspond to any parasite in the gold standard. Sensibility is defined as TP divided by the number of parasites in the Gold Standard, so it refers to the probability of volunteers finding all the malaria parasites in a sample. Specificity defined as TP divided by the total number of clicks (TP+FP) the player made in the sample. It allows representing the probability of not diagnosing malaria in healthy patients. Accuracy, a parameter used by [9], measures TP minus FP over the total number of parasites for the given Gold Standard-image.

\section{Results}

We show the results of the analysis of the performance of individual players. The overall values for sensibility, specificity, and accuracy of the individual gameplays for the 38 images are $0.82,0.60$, and 0.29 respectively. Since difficulty does not change with levels, in higher levels better performance is expected because players might have gained more experience detecting parasites. Figure 3 displays the average accuracy by level. Accuracy appears to be a stable parameter. Although we analyzed individual performance instead of the aggregated one, this finding is consistent with the conclusion of the previous study conducted by [9], that is, starting from the fourth level on, the collective detection of parasites becomes stable. 


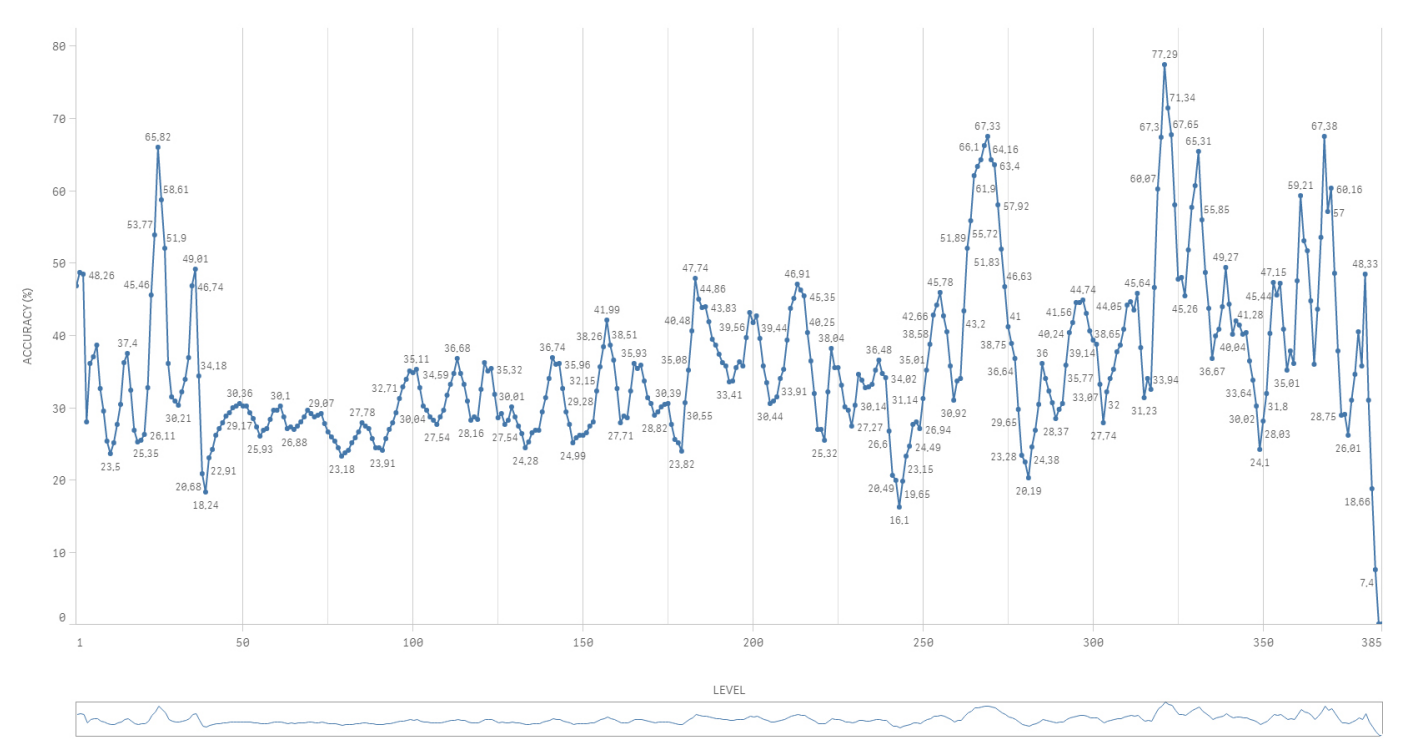

Figure 3. Accuracy per level.

In MalariaSpot, all the images are presented in the same game conditions. Images are shown multiple times to different players. In Figure 4 we sorted all the images into three groups depending on the number of times they have been analyzed by players. Mean values for sensibility and specificity are represented versus the number of times players have analyzed the same image. Mean sensibility and specificity are stable in images with more than 100 analyses.

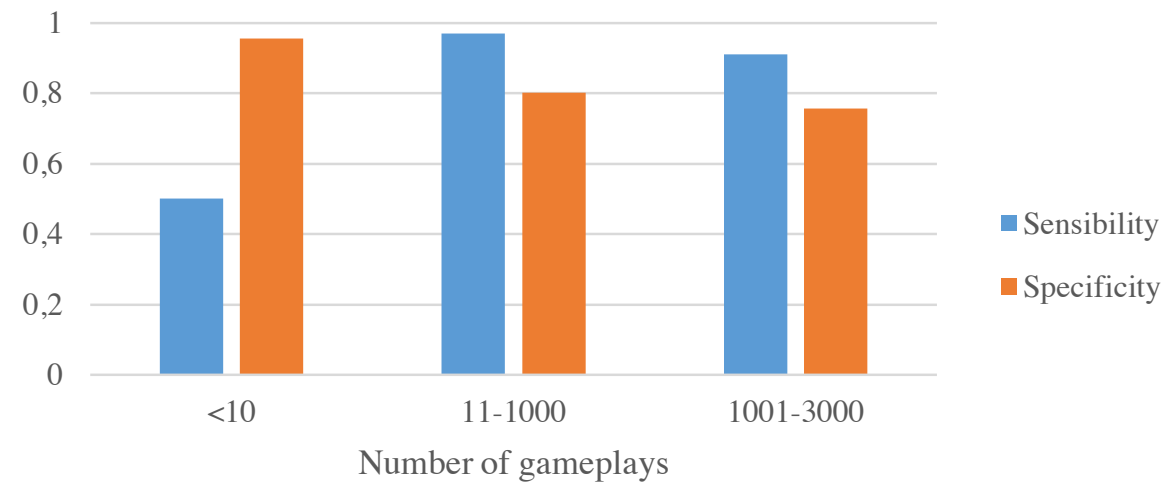

Figure 4. Sensibility and specificity vs the number of gameplays with images.

Figure 5 displays players' performance based on the number of parasites in an image. The diagram shows that sensibility and specificity are proportional to the number of parasites in an image, except for the images with very high parasitaemia ( $>30$ parasites). High presence of parasites in an image makes it easier for players to detect them as their structures tend to look alike and can be compared. However, when there are too many parasites in the image is not possible to tag them all in the allocated time and there is a decrease in sensibility. On the other hand, the most complicate cases are images with very few parasites, as it is hard to find them and there is no reference pattern. 


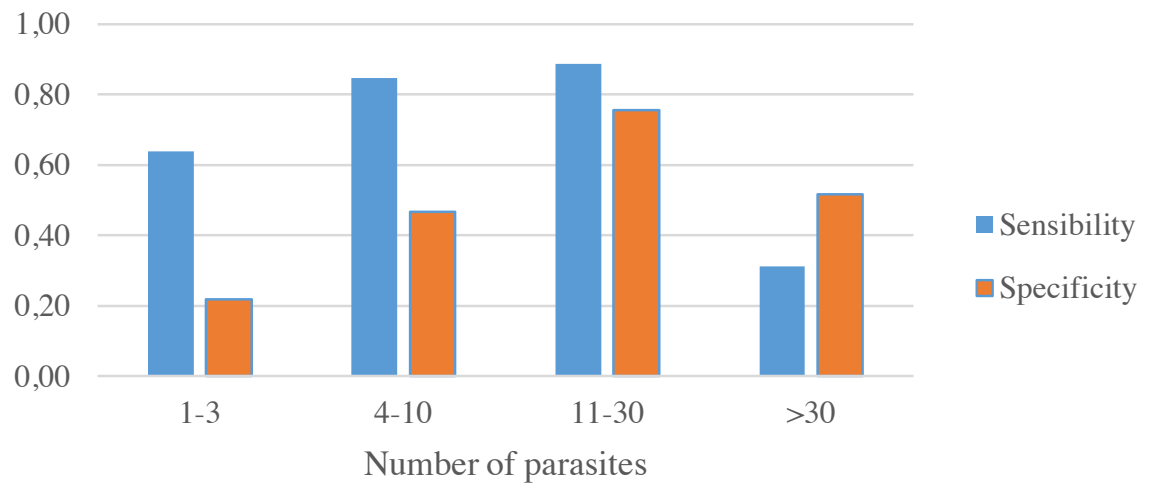

Figure 5. Sensibility and specificity vs number of parasites in the image.

Figures 6 and 7 show how the nature of image of the sample influences players' performance. The quality of images can be determined by multiple variables, but in this study, we evaluated only the means used to capture images (integrated cameras in the microscope vs mobile phones adapted to the microscope ocular) and the origin of the blood smears.

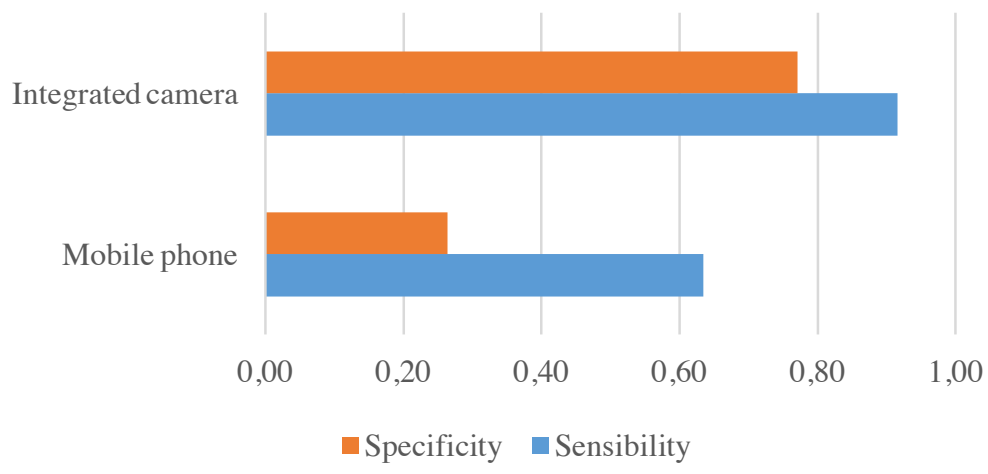

Figure 6. Sensibility and specificity vs the technologies used to acquire images

Data quality is considerably lower in images acquired with mobile phones compared to those digitized with standardized digitalization technology integrated in the microscope. We see that specificity drops more than sensibility. One main difference between these two types of images is the focus, because the structures of parasites are better defined in the images taken with standard integrated digitalization technology. The increase of FP in the images acquired with mobile phones may be the results of this lack of focus. 


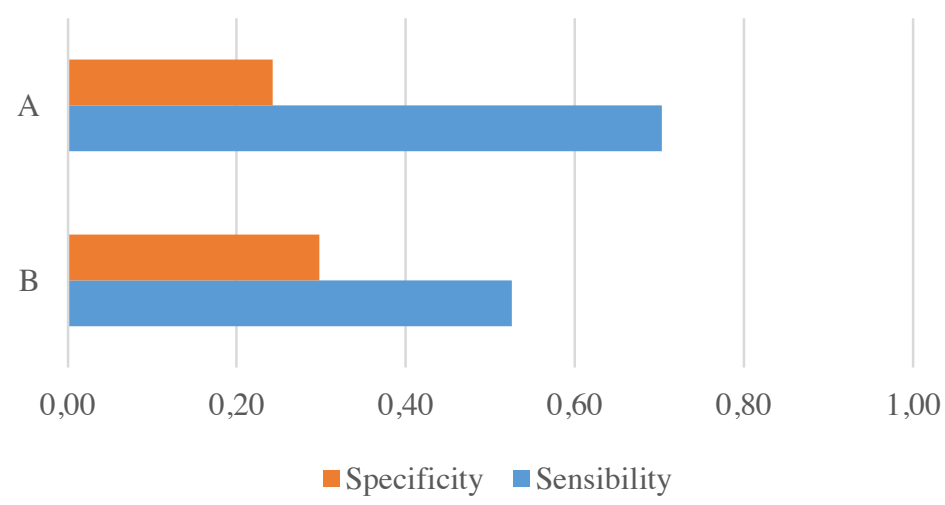

Figure 7. Sensibility and specificity of two image datasets (A, B) digitalized with a mobile phone camera.

Both image datasets from A (Madrid) and B (Mozambique) were acquired with mobile phones. Even though protocols for preparing a sample are internationally standardized, some differences between these two types of images are apparent. Possible differences include the time elapsed since the samples were prepared, the ability of the technician preparing the slide, weather, and quality of dyes.

Regarding the performance of a player over time, Figure 8 represents the accuracy of one individual player who was presented the same image in different game sessions. Game session sequence is the time from opening to closing the application on a mobile phone. Every contiguous gameplay with the same color have been played in the same game session or sequence. In 11 out of the 14 gameplays, this specific player kept or improved his/her results compared to the previous gameplay.

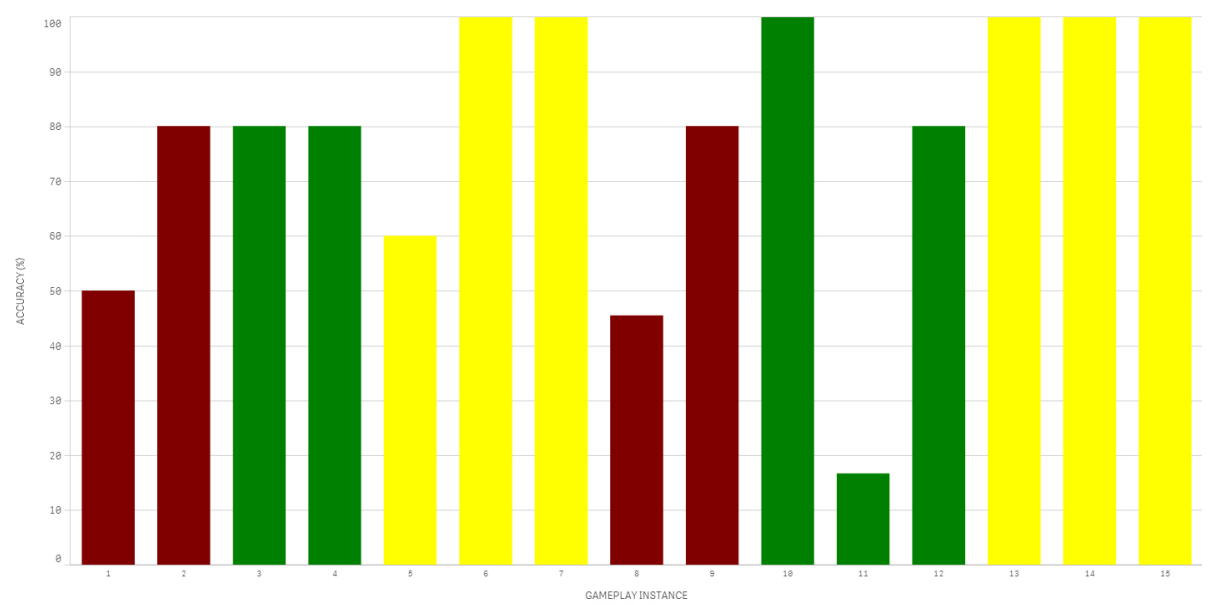

Figure 8. Results of player no. 1 for image no. 4 chronologically sorted.

\section{Discussion}

\section{Principal Findings}

This evaluation indicates that data quality of individual players is influenced by the game design (1-minute game), the technologies for image acquisition, and the origin of the samples. 
In relation to the game design, the presence of high parasitaemia in an image affects detection and can hamper data quality. Players must be able to scan the image and identify as many parasites as possible within a very tight time constraint. Interestingly, finding the exact number of parasites is not medically relevant for cases of high parasitaemia. Under these circumstances, time becomes a critical factor influencing accuracy of detection, arguably more than the level achieved by a player. This design feature is closely connected to the type of task. MalariaSpot is a microtask game and is designed to be inclusive and engage a broad range of citizens. Unlike knowledgeintensive games such as Foldit or The Cure, where players need to invest a substantial amount of time learning the game, MalariaSpot players need visual recognition and speed to play the game and can achieve good results after few gameplays. As already found by [9], this study also suggests that players can improve the needed skills in very few games, as they become better at detecting parasites in blood smears. Being a casual game, MalariaSpot taps into people's passion for online games and not into any specific group of people and practice, such as eBird, which is a mecca for birders worldwide.

The result also indicates that the nature of the input data and the technologies used to acquire images also affect data quality. Different types of images turn in different values of sensibility and specificity, which, in turn, are critical to generate more accurate and usable data. This aspect has substantive implications for the most appropriate choice of technologies for image acquisition.

As found in other citizen science projects [19], MalariaSpot also uses several mechanisms to ensure data quality and validation and counterbalance game design constraints. Such mechanisms include repeated samples/tasks, expert review in the form of the malaria diagnosis protocol, and initial player training. Repeated sample/task by multiple participants is a typical approach in crowdsourcing and is very useful to compare the performances of individual participants and identify quality issues which are likely to be real as they are confirmed by more than one participant.

The use of expert review in the form of the malaria diagnosis protocol counterbalances the time constraint which affects detection in images with high parasitaemia, because this protocol does not require counting each parasite in images including more than 10 parasites. As for initial player training, the use of known images already classified by expert microscopists is a form of expert-trained classification, which is also well-known in crowdsourcing and can be effective to train participants to complete tasks with limited scope, as in MalariaSpot.

\section{Limitations}

Based on the unique sample framework, the results from the study of this specific casual videogame may not be applied to other GWAPs. Performance and participation of players may be biased by players' characteristics, such as, age, gender, level of education, for which we have no data available. There might be multiple external factors that result in the different quality of images coming from the preparations of samples and that impact the difficulty of the task. Further research should be done to analyze in detail the impact of factors affecting individual performance on the collective output of the MalariaSpot system- an algorithm that uses artificial intelligence to combine information from several players in the same image. 


\section{Conclusions}

GWAPs are an appealing form for citizen science, but ensuring data quality can be an issue. We have shown that in MalariaSpot, individual player performance is affected by multiple factors, including those intrinsic to the game - such as the time allowed to complete the analysis of one sample- and those dependent on the nature of the images included in the game- such as the technology used to digitalize the blood samples.

\section{Conflicts of Interest}

None declared

Abbreviations

FP: False Positives

TP: True Positives

\section{Acknowledgments}

This research is supported through funding from Marcus and Marianne Wallenberg grant no. 2013.0020 and from LETStudio at the University of Gothenburg. This work has been partially funded by projects COOP-XV-17 and COOP-XVI-14 from the Direction of Cooperation and Development at the Universidad Politécnica de Madrid and Amazon Web Services research grant to the MalariaSpot project at the Universidad Politecnica de Madrid. Special thanks to the MalariaSpot players.

\section{Authors' Contributions}

Igor Stankovic, María Postigo Camps, Miguel A. Luengo-Oroz, and Daniel Cuadrado Sánchez performed data analysis and interpreted the results. Marisa Ponti, Igor Stankovic, María Postigo Camps and Miguel A. Luengo-Oroz conceived the experiments and wrote the manuscript.

\section{References}

1. von Ahn L, Dabbish L. Designing games with a purpose. Commun. ACM 2008 Aug 01;51(8):57-67. [CrossRef]

2. Bonney R, Shirk JL, Phillips TB, Wiggins A, Ballard HL, Miller-Rushing AJ, Parrish JK. Next steps for citizen science. Science 2014; 343 (6178): 1426 1437. [doi: 10.1126/science.1251554] [PMID: 24675940]

3. Ponciano L, Brasileiro F. Finding volunteers' engagement profiles in human computation for citizen science projects. Human Computation 2014; 1(2): 245264. [doi: 10.15346/hc.v1i2.12]

4. Tinati R, Luczak-Roesch M, Simperl E, Hall W. "Because science is awesome": Studying participation in a citizen science game. Proceedings of WebSci '16; 2016 May22-25; Hannover, Germany. [doi: http://dx.doi.org/10.1145/2908131.2908151]

5. Greenhill A, Holmes K, Woodcock J, Lintott C, Simmons BD, Graham G, Cox J, Young Oh E, Masters K. Playing with science: Exploring how game activity motivates users participation on an online citizen science platform. Aslib Journal of Information Management 2016:68(3): 306 - 325. [doi: 10.1108/AJIM-112015-0182]

6. Prestopnik N, Crowston K, Wang, J. Exploring data quality in games with a purpose. Proceedings of iConference 2014: 213-228. [doi: 10.9776/14066] 
7. Prestopnik N, Crowston K, Wang, J. Gamers, citizen scientists, and data: Exploring participant contributions in two games with a purpose. Computers in Human Behavior 2017 (68): 254-268. [doi: 10.1016/j.chb.2016.11.035]

8. Hunter J, Alabri A, Van Ingen C. Assessing the quality and trustworthiness of citizen science data. Concurrency and Computation: Practice and Experience 2013; 25: 454-466.

9. Luengo-Oroz MA, Arranz A, Frean J. Crowdsourcing malaria parasite quantification: An online game for analyzing images of infected thick blood smears. J Med Internet Res. 2012 Nov 29;14(6):e167. [doi: 10.2196/jmir.2338] [Medline: 23196001]

10. Good BM, Loguercio S, Griffith OL, Nanis M, Wu C, Su AI. The Cure: design and evaluation of a crowdsourcing game for gene selection for breast cancer survival prediction. JMIR Serious Games 2014;2(2):e7 [doi: 10.2196/games.3350] [PMID: 25654473]

11. Khare R, Good BM, Leaman R, Su AI, Lu Z. Crowdsourcing in biomedicine: challenges and opportunities. Briefings in Bioinformatics 2016; 17(1):23-32. [doi: 10.1093/bib/bbv021].

12. Lafourcade M, Joubert A, le Brun, N (2015). Games with a purpose (GWAPs). London and Hoboken, USA: ISTE and Wiley.

13. Good, BM, Su, AI. Crowdsourcing for bioinformatics. Bioinformatics. 2013 Aug 15;29(16):1925-33. [doi: 10.1093/bioinformatics/btt333] [Medline: 23782614]

14. Crowston K, Prestopnik N. Motivation and data quality in a citizen science game: A design science evaluation. Proceedings of the $201346^{\text {th }}$ Hawaii International Conference on System Sciences 2013:450-459. [doi:10.1109/HICSS.2013.413]

15. Crall AW, Newman GJ, Stohlgren TJ, Holfelder KA, Graham J, Waller DM. 2011. Assessing citizen science data quality: an invasive species case study. Conservation Letters 2011 4:433-442. [doi: 10.1111/j.1755263X.2011.00196.x]

16. Jordan RC, Ballard HL, Phillips TB. Key issues and new approaches for evaluating citizen-science learning outcomes. Frontiers in Ecology and the Environment 2012 10:307-309. [doi:10.1890/110280]

17. Lukyanenko R, Parsons J, Wiersma YF. Emerging problems of data quality in citizen science. Conservation Biology 2016; 30: 447-449. [doi:10.1111/cobi.12706].

18. WHO Global Malaria Programme. World Malaria Report 2011. Geneva, Switzerland: World Health Organization; 2011. URL: http://www.who.int/malaria/world_malaria_report_2011/9789241564403_eng.p df [accessed 2017-01-23] [WebCite Cache]

19. Wiggins A, Newman G, Stevenson RD, Crowston K. Mechanisms for data quality and validation in citizen science. 2011; Proceedings of the 2011 IEEE Seventh International Conference on e-Science Workshops; December 5-8. 2011: 14-19 [0.1109/eScienceW.2011.27]

20. Cooper S. A framework for scientific discovery through video games. 2014. New York: ACM and Morgan \& Claypool. [doi: 10.1145/2625848]

21. Lee J, Kladwang W, Lee M, Cantu, D et al. RNA design rules from a massive open laboratory. 2014. Proc. Natl. Acad. Sci. USA, 111:2122-2127.

[doi:10.1073/pnas.1313039111] 
22. Kawrykow A, Roumanis G, Kam A et al. Phylo: A citizen science approach for improving multiple sequence alignment. 2012. PloS One, 2(7):e31362.

[doi:10.1371/journal.pone.0031362] 